\title{
Editorial
}

\section{Special Issue on Brillouin Scattering and Optomechanics}

\author{
Vincent Laude * (D), Jean-Charles Beugnot (D) and Thibaut Sylvestre (iD \\ Institut FEMTO-ST, Université Bourgogne Franche-Comté, CNRS, 25030 Besançon, France; \\ jean-charles.beugnot@femto-st.fr (J.-C.B.); thibaut.sylvestre@femto-st.fr (T.S.) \\ * Correspondence: vincent.laude@femto-st.fr; Tel.: +33-363-082-457
}

Received: 19 August 2019; Accepted: 4 September 2019; Published: 8 September 2019

check for updates

Keywords: Brillouin light scattering; optomechanics; phoxonic crystals; nanoscale optical waveguides

\section{Introduction}

The science of the interaction of sound and light, including acousto-optics and opto-acoustics, has recently witnessed the emergence of new topics and directions that lead to a renewed understanding of fundamental effects and to novel applications. Optomechanical structures, including phoxonic crystals, also known as simultaneous photonic and phononic crystals, are presently being investigated in order to obtain very efficient interactions in tiny volumes. Optical forces, for instance the optical pressure exerted by light on a dielectric micro- or nano-structure, can set a mechanical resonator into motion. They thus allow for the control of phonons with photons, but also for ultimate sensing applications. Concurrently, opto-acoustical interactions in micro- and nano-scale optical fibers and waveguides are being seen in a new light thanks to new materials and structures, leading to a renewed view of Brillouin light scattering.

\section{Optomechanical Structures}

One of the basic questions of optomechanics is how to identify phononic structures that are suitable for enhanced interaction of light and sound. One-dimensional phononic crystals have been studied in this respect, as they allow for rather large amplitude motion and can be fabricated at a scale commensurate with a light wavelength. Gueddida et al. [1] considered deeply grooved gratings of nanoridges fabricated in epoxy on a glass substrate. They identified various acoustic phonon modes of the one-dimensional phononic crystal. One interesting possibility is to fill the nanoridge structure with a viscous liquid, thus producing hybrid acousto-elastic modes that propagate along the 1D finite height multilayer array and can be used for sensing applications.

Another important direction is that of phonon resonators presenting very low loss, since vibrations can be trapped and oscillate for long times, with important applications to ultrastable clocks. Goryachev et al. [2] studied a quartz crystal Bulk Acoustic Wave (BAW) resonator that is immune to suspension losses and so exhibits an extremely high acoustic $\mathrm{Q}$-factor at low temperature. They coupled the resonator to a Superconducting Quantum Interference Device (SQUID) amplifier and investigated the nonlinear effects. They found the nonlinearity to provide quasi-periodic spectra in both incident power and frequency. Their results are steps toward future cryogenic quartz oscillators in the strong signal regime.

A recent direction of research is that of topological wave systems, in both photonics and phononics. Topological protection can be obtained in certain passive structures based on symmetry considerations. Esmann and Lanzillotti-Kimura [3] considered nanofabricated Fabry-Perot resonators operating with phonons in the GHz-THz range. They drew a parallel between the Su-Schrieffer-Heeger (SSH) model, 
which is a paradigm that accounts for the topological properties of many one-dimensional structures, and the standard Distributed Bragg Reflector (DBR)-based acoustic Fabry-Perot interferometers. They explained the existence of confined modes in a Fabry-Perot cavity in terms of the symmetry inversion of Bloch modes at the Brillouin zone edge.

\section{Brillouin Light Scattering}

Brillouin light scattering in the bulk of transparent media or at the surface of opaque media is an established technique to probe acoustic phonons. Carlotti [4] reviewed experimental methods to achieve the elastic characterization of thin films, multilayers, and acoustic resonators operating in the $\mathrm{GHz}$ range of frequencies, with a particular stress on surface Brillouin Light Scattering (surf-BLS). Interestingly, the recently-developed micro-focused scanning version of the surf-BLS technique opened new opportunities for the imaging of acoustic fields in resonators and in artificially-patterned acoustic metamaterials.

In optical fibers and waveguides, much progress has been made recently toward nanoscale diameters where light and sound are simultaneously strongly confined. Brillouin light scattering experiments either rely on the diffraction of light waves by random acoustic phonons, originating from thermal fluctuations, or by coherent acoustic waves generated by a transducer or from the mixing of two optical waves. In both cases, it is usually found that the measured spectra are similar. Laude and Beugnot [5] examined the origin of the similarity by discussing the structure of the elastodynamic equation. They argued that the spectral response was determined by pre-existing phonon solutions, having given intrinsic loss, under either a stochastic Langevin excitation or in response to a coherent optical mixing force.

Author Contributions: conceptualization, V.L., J.-C.B. and T.S.; writing-original draft preparation, V.L.; writing-review and editing, J.-C.B. and T.S.

Funding: This research received no external funding.

Acknowledgments: The Special Issue on Brillouin scattering and optomechanics would not be possible without the contributions of various talented authors, hardworking and professional reviewers, and the dedicated editorial team of Applied Sciences. We express our gratitude to the editorial team of Applied Sciences and our special thanks to Daria Shi.

Conflicts of Interest: The authors declare no conflict of interest.

\section{References}

1. Gueddida, A.; Pennec, Y.; El Boudouti, E.H.; Fytas, G.; Djafari Rouhani, B. Propagation of Elastic Waves in a One-Dimensional High Aspect Ratio Nanoridge Phononic Crystal. Appl. Sci. 2018, 8, 805. [CrossRef]

2. Goryachev, M.; Ivanov, E.N.; Galliou, S.; Tobar, M.E. Inducing Strong Non-Linearities in a Phonon Trapping Quartz Bulk Acoustic Wave Resonator Coupled to a Superconducting Quantum Interference Device. Appl. Sci. 2018, 8, 602. [CrossRef]

3. Esmann, M.; Lanzillotti-Kimura, N.D. A Topological View on Optical and Phononic Fabry-Perot Microcavities through the Su-Schrieffer-Heeger Model. Appl. Sci. 2018, 8, 527. [CrossRef]

4. Carlotti, G. Elastic Characterization of Transparent and Opaque Films, Multilayers and Acoustic Resonators by Surface Brillouin Scattering: A Review. Appl. Sci. 2018, 8, 124. [CrossRef]

5. Laude, V.; Beugnot, J.-C. Spontaneous Brillouin Scattering Spectrum and Coherent Brillouin Gain in Optical Fibers. Appl. Sci. 2018, 8, 907, do:10.3390/app8060907. [CrossRef] 